(n)

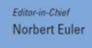
Journal of Nonlinear Mathematical Physics

\title{
A Vector Fokas-Lenells System from the Coupled Nonlinear Schro̊dinger Equations
}

MengXia Zhang, ShaoLing He, ShuQiang Lv

To cite this article: MengXia Zhang, ShaoLing He, ShuQiang Lv (2015) A Vector FokasLenells System from the Coupled Nonlinear Schrödinger Equations, Journal of Nonlinear Mathematical Physics 22:1, 144-154, DOI:

https://doi.org/10.1080/14029251.2015.996445

To link to this article: https://doi.org/10.1080/14029251.2015.996445

Published online: 04 January 2021 


\title{
A Vector Fokas-Lenells System from the Coupled Nonlinear Schrödinger Equations
}

\author{
MengXia Zhang \\ Department of Mathematics, China University of Mining and Technology, \\ Beijing, 100083, the People's Republic of China \\ zmx@cumtb.edu.cn \\ ShaoLing $\mathrm{He}$ \\ LiYun Experimental School of Beijing Normal University, \\ Beijing, 100014, the People's Republic of China \\ heshaoling86818@163.com \\ ShuQiang Lv \\ Department of Basic Education, College of Applied Arts and Science of Beijing Union University, \\ Beijing 100191, the People's Republic of China \\ shuqianglv@163.com
}

Received 29 July 2014

Accepted 23 October 2014

\begin{abstract}
With the aid of the spectral gradient method of Fuchssteiner, the compatible pair of Hamiltonian operators for the coupled NLS hierarchy is rediscovered. This result enables us to construct a hierarchy, which contains a vector generalization of Fokas-Lenells system. The vector Fokas-Lenells system is shown to be bi-Hamiltonian and to possess a Lax pair.
\end{abstract}

Keywords: Hamiltonian operators; Lax pair; coupled nonlinear Schrödinger equation; derivative nonlinear Schrödinger equation.

2000 Mathematics Subject Classification: 37K10, 35Q55

\section{Introduction}

The nonlinear Schrödinger (NLS) equation is one of the most important equations in both mathematics and physics. On the one hand, it has numerous applications in physics such as water waves, optics and plasma physics. On the other hand, the NLS equation has been playing a key role in the development of the soliton theory or the theory of modern integrable systems (see Ref. [1] and the references therein).

The NLS equation has been generalized in various different ways. One particular extension, constructed by Manakov in the seventies of the last century in [20], is the coupled NLS or Manakov model. This system reads as

$$
\begin{aligned}
& q_{1 t}=i\left(q_{1 x x}+\kappa\left(\left|q_{1}\right|^{2}+\left|q_{2}\right|^{2}\right) q_{1}\right), \\
& q_{2 t}=i\left(q_{2 x x}+\kappa\left(\left|q_{1}\right|^{2}+\left|q_{2}\right|^{2}\right) q_{2}\right),
\end{aligned}
$$

where $\kappa$ is a real parameter. Like the NLS equation itself, the coupled NLS system also is physically relevant and has been studied extensively (see Refs. [2] and [27] for examples). 
A more recent generalization of the NLS equation is the following one

$$
i u_{t}-v u_{t x}+\gamma u_{x x}+\sigma|u|^{2}\left(u+i v u_{x}\right)=0, \quad \sigma= \pm 1,
$$

which depends on two real parameters $\gamma$ and $v$. Above equation was discovered in 1995 by Fokas [6] and was rederived by Lenells as a model for nonlinear pulse propagation in certain fibres [15]. This equation, referred as Fokas-Lenells (FL) equation, was studied by Fokas and Lenells $[16,17]$ and others (see Refs. [12, 21, 22, 26, 28] for examples). It is remarked that the bi-Hamiltonian method used by Fokas originated from a fruitful idea of Fokas and Fuchssteiner [8] and has been applied successfully to a number of integrable equations (see Refs. [11,24]).

The aim of the present paper is to propose a generalization of the coupled NLS equation or Manakov system. We will start with a more general spectral problem or the coupled AKNS spectral problem, which is of the constrained KP type [4, 5, 13, 14, 25]. The bi-Hamiltonian structure for the hierarchy associated with the coupled AKNS spectral problem is known $[3,9,19]$, but for our purpose of constructing integrable systems we will rederive them by means of the spectral gradient method of Fuchssteiner [10]. This will be done in the next section. In section 3, we will take up the bi-Hamiltonian method to produce an integrable hierarchy, which includes a generalized Manakov system or vector FL system as a particular flow. In section 4, we will work out a Lax representation for the vector FL system.

\section{Spectral Gradient Method and Coupled AKNS Spectral Problem}

We consider the following spectral problem

$$
\left(\begin{array}{l}
\phi_{1} \\
\phi_{2} \\
\phi_{3}
\end{array}\right)_{x}=\left(\begin{array}{ccc}
2 \lambda & q_{1} & q_{2} \\
r_{1} & -\lambda & 0 \\
r_{2} & 0 & -\lambda
\end{array}\right)\left(\begin{array}{l}
\phi_{1} \\
\phi_{2} \\
\phi_{3}
\end{array}\right),
$$

where $\lambda$ is the spectral parameter, $q_{i}, r_{i}(i=1,2)$ are field variables depending on $x$ and $t$. This spectral problem (2.1) is the matrix version of

$$
\left(\partial-q_{1} \partial^{-1} r_{1}-q_{2} \partial^{-1} r_{2}\right) \varphi=3 \lambda \varphi,
$$

a spectral problem of the constrained KP type [19].

In fact, we introduce the following new variables by

$$
\varphi=\varphi_{1}, \partial^{-1} r_{1} \varphi=\varphi_{2}, \partial^{-1} r_{2} \varphi=\varphi_{3},
$$

then the Eq. (2.2) becomes

$$
\left(\begin{array}{l}
\varphi_{1} \\
\varphi_{2} \\
\varphi_{3}
\end{array}\right)_{x}=\left(\begin{array}{ccc}
3 \lambda & q_{1} & q_{2} \\
r_{1} & 0 & 0 \\
r_{2} & 0 & 0
\end{array}\right)\left(\begin{array}{l}
\varphi_{1} \\
\varphi_{2} \\
\varphi_{3}
\end{array}\right)
$$

Through a simple gauge transformation, namely

$$
\varphi_{1}=e^{\lambda x} \phi_{1}, \varphi_{2}=e^{\lambda x} \phi_{2}, \varphi_{3}=e^{\lambda x} \phi_{3},
$$

then we arrive at the Eq. (2.1). 
The integrable systems associated with (2.1) and their bi-Hamiltonian structure are studied $[19,25]$, which include the celebrated coupled NLS equation or Manakov system [20] as a particular flow. For our purpose, we adopt Fuchssteiner's spectral gradient method [10] and rederive the recursion operator and bi-Hamiltonian structure. To this end, we need the adjoint problem of Eq. (2.1), which reads as

$$
\left(\psi_{1}, \psi_{2}, \psi_{3}\right)_{x}=-\left(\psi_{1}, \psi_{2}, \psi_{3}\right)\left(\begin{array}{ccc}
2 \lambda & q_{1} & q_{2} \\
r_{1} & -\lambda & 0 \\
r_{2} & 0 & -\lambda
\end{array}\right)
$$

The directional derivative of $\left(\phi_{1}, \phi_{2}, \phi_{3}\right)^{T}$ determined by Eq. (2.1) in the direction $q_{1}+\varepsilon Q$ is given by

$$
\begin{aligned}
& \left(\begin{array}{l}
\phi_{1}^{\prime}[Q] \\
\phi_{2}^{\prime}[Q] \\
\phi_{3}^{\prime}[Q]
\end{array}\right)_{x}=\left(\begin{array}{ccc}
2 \lambda & q_{1} & q_{2} \\
r_{1} & -\lambda & 0 \\
r_{2} & 0 & -\lambda
\end{array}\right)\left(\begin{array}{l}
\phi_{1}^{\prime}[Q] \\
\phi_{2}^{\prime}[Q] \\
\phi_{3}^{\prime}[Q]
\end{array}\right) \\
& +\left(\begin{array}{ccc}
2<Q, \lambda_{q_{1}}> & Q & 0 \\
0 & -<Q, \lambda_{q_{1}}> & 0 \\
0 & 0 & -<Q, \lambda_{q_{1}}>
\end{array}\right)\left(\begin{array}{l}
\phi_{1} \\
\phi_{2} \\
\phi_{3}
\end{array}\right),
\end{aligned}
$$

where $\langle\cdot, \cdot\rangle$ denotes the paring between tangent vectors and cotangent vectors, and is defined by

$$
<\rho, \sigma>=\int_{-\infty}^{+\infty} \rho(x) \sigma(x) d x .
$$

Left multiplying Eq. (2.5) by $\left(\psi_{1}, \psi_{2}, \psi_{3}\right)$, then integrating over $x$ from $-\infty$ to $\infty$, we obtain

$$
\begin{array}{r}
\int_{-\infty}^{+\infty}\left(\psi_{1}, \psi_{2}, \psi_{3}\right)\left(\begin{array}{l}
\phi_{1}^{\prime}[Q] \\
\phi_{2}^{\prime}[Q] \\
\phi_{3}^{\prime}[Q]
\end{array}\right) d x=\int_{-\infty}^{+\infty}\left(\psi_{1}, \psi_{2}, \psi_{3}\right)\left(\begin{array}{ccc}
2 \lambda & q_{1} & q_{2} \\
r_{1} & -\lambda & 0 \\
r_{2} & 0 & -\lambda
\end{array}\right)\left(\begin{array}{l}
\phi_{1}^{\prime}[Q] \\
\phi_{2}^{\prime}[Q] \\
\phi_{3}^{\prime}[Q]
\end{array}\right) d x \\
+\int_{-\infty}^{+\infty}\left(\psi_{1}, \psi_{2}, \psi_{3}\right)\left(\begin{array}{ccc}
2<Q, \lambda_{q_{1}}> & Q & 0 \\
0 & -<Q, \lambda_{q_{1}}> & 0 \\
0 & 0 & -<Q, \lambda_{q_{1}}>
\end{array}\right)\left(\begin{array}{l}
\phi_{1} \\
\phi_{2} \\
\phi_{3}
\end{array}\right) d x .
\end{array}
$$

Assuming that $\left(\psi_{1}, \psi_{2}, \psi_{3}\right)$ solves the adjoint problem (2.4) and integrating the left-hand-side of the above equation by parts, we find

$$
\int_{-\infty}^{+\infty}\left(\psi_{1}, \psi_{2}, \psi_{3}\right)\left(\begin{array}{ccc}
2<Q, \lambda_{q_{1}}> & Q & 0 \\
0 & -<Q, \lambda_{q_{1}}> & 0 \\
0 & 0 & -<Q, \lambda_{q_{1}}>
\end{array}\right)\left(\begin{array}{l}
\phi_{1} \\
\phi_{2} \\
\phi_{3}
\end{array}\right) d x=0,
$$

or equivalently,

$$
<Q, \lambda_{q_{1}}>\int_{-\infty}^{+\infty}\left(2 \psi_{1} \phi_{1}-\psi_{2} \phi_{2}-\psi_{3} \phi_{3}\right) d x+\int_{-\infty}^{+\infty} Q \psi_{1} \phi_{2} d x=0
$$

which holds for arbitrary $Q$. Thus, we obtain the gradient of $\lambda$ with respect to $q_{1}$, namely

$$
\lambda_{q_{1}}=-\frac{\psi_{1} \phi_{2}}{\int_{-\infty}^{+\infty}\left(2 \psi_{1} \phi_{1}-\psi_{2} \phi_{2}-\psi_{3} \phi_{3}\right) d x} .
$$


Similarly, we find the gradients of $\lambda$ with respect to $q_{2}, r_{1}, r_{2}$, and they read as

$$
\begin{aligned}
& \lambda_{q_{2}}=-\frac{\psi_{1} \phi_{3}}{\int_{-\infty}^{+\infty}\left(2 \psi_{1} \phi_{1}-\psi_{2} \phi_{2}-\psi_{3} \phi_{3}\right) d x}, \\
& \lambda_{r_{1}}=-\frac{\psi_{2} \phi_{1}}{\int_{-\infty}^{+\infty}\left(2 \psi_{1} \phi_{1}-\psi_{2} \phi_{2}-\psi_{3} \phi_{3}\right) d x}, \\
& \lambda_{r_{2}}=-\frac{\psi_{3} \phi_{1}}{\int_{-\infty}^{+\infty}\left(2 \psi_{1} \phi_{1}-\psi_{2} \phi_{2}-\psi_{3} \phi_{3}\right) d x} .
\end{aligned}
$$

Since we are interested in constructing an eigenvalue problem for the gradients, the constant coefficients may be omitted, namely

$$
\left(\begin{array}{l}
\lambda_{q_{1}} \\
\lambda_{q_{2}} \\
\lambda_{r_{1}} \\
\lambda_{r_{2}}
\end{array}\right) \propto\left(\begin{array}{l}
\psi_{1} \phi_{2} \\
\psi_{1} \phi_{3} \\
\psi_{2} \phi_{1} \\
\psi_{3} \phi_{1}
\end{array}\right) .
$$

Thus we have

Theorem 2.1. The gradients of the spectral parameter in the coupled AKNS hierarchy satisfy the equation

$$
\begin{gathered}
\left(\begin{array}{cccc}
2 q_{1} \partial^{-1} q_{1} & q_{1} \partial^{-1} q_{2}+q_{2} \partial^{-1} q_{1} & R_{1} & -q_{1} \partial^{-1} r_{2} \\
q_{2} \partial^{-1} q_{1}+q_{1} \partial^{-1} q_{2} & 2 q_{2} \partial^{-1} q_{2} & -q_{2} \partial^{-1} r_{1} & R_{2} \\
-\left(R_{1}\right)^{*} & -r_{1} \partial^{-1} q_{2} & 2 r_{1} \partial^{-1} r_{1} & r_{1} \partial^{-1} r_{2}+r_{2} \partial^{-1} r_{1} \\
-r_{2} \partial^{-1} q_{1} & -\left(R_{2}\right)^{*} & r_{2} \partial^{-1} r_{1}+r_{1} \partial^{-1} r_{2} & 2 r_{2} \partial^{-1} r_{2}
\end{array}\right)\left(\begin{array}{l}
\lambda_{q_{1}} \\
\lambda_{q_{2}} \\
\lambda_{r_{1}} \\
\lambda_{r_{2}}
\end{array}\right) \\
=3 \lambda\left(\begin{array}{cccc}
0 & 0 & 1 & 0 \\
0 & 0 & 0 & 1 \\
-1 & 0 & 0 & 0 \\
0 & -1 & 0 & 0
\end{array}\right)\left(\begin{array}{l}
\lambda_{q_{1}} \\
\lambda_{q_{2}} \\
\lambda_{r_{1}} \\
\lambda_{r_{2}}
\end{array}\right)
\end{gathered}
$$

where $R_{1}$ and $R_{2}$ are defined by

$$
R_{1}=\partial-2 q_{1} \partial^{-1} r_{1}-q_{2} \partial^{-1} r_{2}, R_{2}=\partial-2 q_{2} \partial^{-1} r_{2}-q_{1} \partial^{-1} r_{1} .
$$

Proof. From Eqs. (2.1) and (2.4), one easily obtains

$$
\begin{aligned}
& \left(\psi_{1} \phi_{2}\right)_{x}=-3 \lambda \psi_{1} \phi_{2}+r_{1}\left(\psi_{1} \phi_{1}-\psi_{2} \phi_{2}\right)-r_{2} \psi_{3} \phi_{2}, \\
& \left(\psi_{1} \phi_{3}\right)_{x}=-3 \lambda \psi_{1} \phi_{3}+r_{2}\left(\psi_{1} \phi_{1}-\psi_{3} \phi_{3}\right)-r_{1} \psi_{2} \phi_{3}, \\
& \left(\psi_{2} \phi_{1}\right)_{x}=3 \lambda \psi_{2} \phi_{1}-q_{1}\left(\psi_{1} \phi_{1}-\psi_{2} \phi_{2}\right)+q_{2} \psi_{2} \phi_{3}, \\
& \left(\psi_{3} \phi_{1}\right)_{x}=3 \lambda \psi_{3} \phi_{1}-q_{2}\left(\psi_{1} \phi_{1}-\psi_{3} \phi_{3}\right)+q_{1} \psi_{3} \phi_{2} .
\end{aligned}
$$

Also, we notice that

$$
\begin{aligned}
\left(\psi_{1} \phi_{1}-\psi_{2} \phi_{2}\right)_{x} & =-2 r_{1} \psi_{2} \phi_{1}-r_{2} \psi_{3} \phi_{1}+2 q_{1} \psi_{1} \phi_{2}+q_{2} \psi_{1} \phi_{3} \\
\left(\psi_{1} \phi_{1}-\psi_{3} \phi_{3}\right)_{x} & =-2 r_{2} \psi_{3} \phi_{1}-r_{1} \psi_{2} \phi_{1}+2 q_{2} \psi_{1} \phi_{3}+q_{1} \psi_{1} \phi_{2}, \\
\left(\psi_{3} \phi_{2}\right)_{x} & =r_{1} \psi_{3} \phi_{1}-q_{2} \psi_{1} \phi_{2} \\
\left(\psi_{2} \phi_{3}\right)_{x} & =r_{2} \psi_{2} \phi_{1}-q_{1} \psi_{1} \phi_{3} .
\end{aligned}
$$


Then substituting Eqs. (2.13)-(2.16) into Eqs. (2.9)-(2.12), we get

$$
\begin{aligned}
& \left(\begin{array}{cccc}
\left(R_{1}\right)^{*} & r_{1} \partial^{-1} q_{2} & -2 r_{1} \partial^{-1} r_{1} & -r_{1} \partial^{-1} r_{2}-r_{2} \partial^{-1} r_{1} \\
r_{2} \partial^{-1} q_{1} & \left(R_{2}\right)^{*} & -r_{2} \partial^{-1} r_{1}-r_{1} \partial^{-1} r_{2} & -2 r_{2} \partial^{-1} r_{2} \\
-2 q_{1} \partial^{-1} q_{1} & -q_{1} \partial^{-1} q_{2}-q_{2} \partial^{-1} q_{1} & -R_{1} & q_{1} \partial^{-1} r_{2} \\
-q_{2} \partial^{-1} q_{1}-q_{1} \partial^{-1} q_{2} & -2 q_{2} \partial^{-1} q_{2} & q_{2} \partial^{-1} r_{1} & -R_{2}
\end{array}\right)\left(\begin{array}{l}
\psi_{1} \phi_{2} \\
\psi_{1} \phi_{3} \\
\psi_{2} \phi_{1} \\
\psi_{3} \phi_{1}
\end{array}\right) \\
& =3 \lambda\left(\begin{array}{cccc}
1 & 0 & 0 & 0 \\
0 & 1 & 0 & 0 \\
0 & 0 & -1 & 0 \\
0 & 0 & 0 & -1
\end{array}\right)\left(\begin{array}{l}
\psi_{1} \phi_{2} \\
\psi_{1} \phi_{3} \\
\psi_{2} \phi_{1} \\
\psi_{3} \phi_{1}
\end{array}\right)
\end{aligned}
$$

which, after left multiplying by the matrix

$$
\left(\begin{array}{cccc}
0 & 0 & -1 & 0 \\
0 & 0 & 0 & -1 \\
-1 & 0 & 0 & 0 \\
0 & -1 & 0 & 0
\end{array}\right)
$$

leads to Eq. (2.7), the eigenvalue problem of the spectral gradients.

The eigenvalue problem (2.7) may be rewritten as

$$
B_{1}\left(\begin{array}{c}
\lambda_{q_{1}} \\
\lambda_{q_{2}} \\
\lambda_{r_{1}} \\
\lambda_{r_{2}}
\end{array}\right)=3 \lambda B_{0}\left(\begin{array}{c}
\lambda_{q_{1}} \\
\lambda_{q_{2}} \\
\lambda_{r_{1}} \\
\lambda_{r_{2}}
\end{array}\right)
$$

where

$$
\begin{gathered}
B_{0}=\left(\begin{array}{cccc}
0 & 0 & 1 & 0 \\
0 & 0 & 0 & 1 \\
-1 & 0 & 0 & 0 \\
0 & -1 & 0 & 0
\end{array}\right), \\
B_{1}=\left(\begin{array}{cccc}
2 q_{1} \partial^{-1} q_{1} & q_{1} \partial^{-1} q_{2}+q_{2} \partial^{-1} q_{1} & R_{1} & -q_{1} \partial^{-1} r_{2} \\
q_{2} \partial^{-1} q_{1}+q_{1} \partial^{-1} q_{2} & 2 q_{2} \partial^{-1} q_{2} & -q_{2} \partial^{-1} r_{1} & R_{2} \\
-\left(R_{1}\right)^{*} & -r_{1} \partial^{-1} q_{2} & 2 r_{1} \partial^{-1} r_{1} & r_{1} \partial^{-1} r_{2}+r_{2} \partial^{-1} r_{1} \\
-r_{2} \partial^{-1} q_{1} & -\left(R_{2}\right)^{*} & r_{2} \partial^{-1} r_{1}+r_{1} \partial^{-1} r_{2} & 2 r_{2} \partial^{-1} r_{2}
\end{array}\right) .
\end{gathered}
$$

Therefore, we recover the compatible Hamiltonian operators $B_{0}$ and $B_{1}$, and the recursion operator $\mathscr{R}=B_{1} B_{0}^{-1}$ for the coupled AKNS hierarchy $[3,9,19]$.

\section{Vector Fokas-Lenells Equation}

Following the idea of Fokas, Fuchssteiner, Olver and Rosenau, we now recombine the Hamiltonian operators found above section so that new compatible Hamiltonian pair is generated. To this end, 
we introduce

$$
\begin{gathered}
\beta_{0}=\left(\begin{array}{cccc}
1 & 0 & 0 & 0 \\
0 & 1 & 0 & 0 \\
0 & 0 & -1 & 0 \\
0 & 0 & 0 & -1
\end{array}\right), \\
\theta_{2}=\left(\begin{array}{cccc}
R_{3} & -q_{1} \partial^{-1} r_{2} & 2 q_{1} \partial^{-1} q_{1} & q_{1} \partial^{-1} q_{2}+q_{2} \partial^{-1} q_{1} \\
-q_{2} \partial^{-1} r_{1} & R_{4} & q_{2} \partial^{-1} q_{1}+q_{1} \partial^{-1} q_{2} & 2 q_{2} \partial^{-1} q_{2} \\
2 r_{1} \partial^{-1} r_{1} & r_{1} \partial^{-1} r_{2}+r_{2} \partial^{-1} r_{1} & -\left(R_{3}\right)^{*} & -r_{1} \partial^{-1} q_{2} \\
r_{2} \partial^{-1} r_{1}+r_{1} \partial^{-1} r_{2} & 2 r_{2} \partial^{-1} r_{2} & -r_{2} \partial^{-1} q_{1} & -\left(R_{4}\right)^{*}
\end{array}\right),
\end{gathered}
$$

where $R_{3}$ and $R_{4}$ are defined by

$$
R_{3}=\gamma \partial-2 q_{1} \partial^{-1} r_{1}-q_{2} \partial^{-1} r_{2}, R_{4}=\gamma \partial-2 q_{2} \partial^{-1} r_{2}-q_{1} \partial^{-1} r_{1} .
$$

We further introduce

$$
\theta_{1}=\beta_{0}+i v I \partial
$$

where $I$ is a $4 \times 4$ identity matrix, and new variables

$$
q_{k}=u_{k}+i v u_{k, x}, \quad r_{k}=v_{k}-i v v_{k, x}, \quad(k=1,2) .
$$

Then we may construct a hierarchy of equations by

$$
\left(\begin{array}{l}
q_{1} \\
q_{2} \\
r_{1} \\
r_{2}
\end{array}\right)_{t_{n}}=\left(i \theta_{2} \theta_{1}^{-1}\right)^{n}\left(\begin{array}{c}
q_{1} \\
q_{2} \\
r_{1} \\
r_{2}
\end{array}\right)_{x}
$$

which in the simplest case reads explicitly as

$$
\left(\begin{array}{l}
q_{1} \\
q_{2} \\
r_{1} \\
r_{2}
\end{array}\right)_{t}=i\left(\begin{array}{c}
\gamma u_{1, x x}-2 q_{1} u_{1} v_{1}-q_{1} u_{2} v_{2}-q_{2} u_{1} v_{2} \\
\gamma u_{2, x x}-2 q_{2} u_{2} v_{2}-q_{2} u_{1} v_{1}-q_{1} u_{2} v_{1} \\
-\gamma v_{1, x x}+2 r_{1} u_{1} v_{1}+r_{1} u_{2} v_{2}+r_{2} u_{2} v_{1} \\
-\gamma v_{2, x x}+2 r_{2} u_{2} v_{2}+r_{2} u_{1} v_{1}+r_{1} u_{1} v_{2}
\end{array}\right)
$$

If we assume

$$
u_{1}=-\tilde{u}, v_{1}=\frac{1}{2} \tilde{v}, u_{2}=v_{2}=0, q_{1}=-\tilde{q}, r_{1}=\frac{1}{2} \tilde{r}
$$

then the Eq. (3.2) reduces to

$$
\left(\begin{array}{c}
\tilde{q} \\
\tilde{r}
\end{array}\right)_{t}=i\left(\begin{array}{c}
\gamma \tilde{u}_{x x}+\tilde{q} \tilde{u} \tilde{v} \\
-\gamma \tilde{v}_{x x}-\tilde{r} \tilde{u} \tilde{v}
\end{array}\right),
$$

which is the equation discussed by Lenells and Fokas [17]. Thus, we name the Eq. (3.2) as the vector Fokas-Lenells (vFL) system and the hierarchy (3.1) as the vFL hierarchy. 
The vFL system (3.2) is a bi-Hamiltonian system by construction. Indeed, let

$$
\beta_{1}=\left(\begin{array}{llll}
0 & 0 & 1 & 0 \\
0 & 0 & 0 & 1 \\
1 & 0 & 0 & 0 \\
0 & 1 & 0 & 0
\end{array}\right)
$$

and define the two Hamiltonian operators

$$
\begin{aligned}
J_{2} & =\theta_{2} \beta_{1}, \\
J_{1} & =-i \theta_{1} \beta_{1} \\
& =\left(\begin{array}{cccc}
0 & 0 & -i+v \partial & 0 \\
0 & 0 & 0 & -i+v \partial \\
i+v \partial & 0 & 0 & 0 \\
0 & i+v \partial & 0 & 0
\end{array}\right) .
\end{aligned}
$$

Then the vFL system may be reformulated as

$$
\left(\begin{array}{c}
q_{1} \\
q_{2} \\
r_{1} \\
r_{2}
\end{array}\right)_{t}=J_{2}\left(\begin{array}{c}
\frac{\delta H_{1}}{\delta q_{1}} \\
\frac{\delta H_{1}}{\delta q_{2}} \\
\frac{\delta H_{1}}{\delta r_{1}} \\
\frac{\delta H_{1}}{\delta r_{2}}
\end{array}\right)=J_{1}\left(\begin{array}{c}
\frac{\delta H_{2}}{\delta q_{1}} \\
\frac{\delta H_{2}}{\delta q_{2}} \\
\frac{\delta H_{2}}{\delta r_{1}} \\
\frac{\delta H_{2}}{\delta r_{2}}
\end{array}\right)
$$

with the Hamiltonian functionals

$$
\begin{aligned}
& H_{1}=-i \int\left(u_{1} r_{1, x}+u_{2} r_{2, x}\right) d x \\
& H_{2}=\frac{1}{2} \int\left(\left(u_{1} v_{1}+u_{2} v_{2}\right)\left(q_{1} v_{1}+q_{2} v_{2}+r_{1} u_{1}+r_{2} u_{2}\right)-2 \gamma\left(u_{1} v_{1, x x}+u_{2} v_{2, x x}\right)\right) d x .
\end{aligned}
$$

By a direct proof, we have proved the Hamiltonian nature of the operators (3.3).

\section{A Lax Pair}

With the aid of the correspondence between the coupled AKNS spectral problem (2.1) and the eigenvalue equation (2.17), one can construct a spectral problem for the vFL system (3.2). Indeed, we consider the following eigenvalue equation related to the recursion operator [7], i.e.,

$$
L^{\dagger} G_{\mu}=i \mu G_{\mu}
$$

where $\dagger$ denotes the formal adjoint of an operator, and $L=J_{2} J_{1}^{-1}=i \theta_{2} \theta_{1}^{-1}$. Then from Eq. (4.1), we obtain

$$
\theta_{2}^{\dagger} G_{\mu}=\mu \theta_{1}^{\dagger} G_{\mu}
$$


where

$$
\begin{aligned}
\theta_{1}^{\dagger} & =\left(\begin{array}{cccc}
1-i v \partial & 0 & 0 & 0 \\
0 & 1-i v \partial & 0 & 0 \\
0 & 0 & -1-i v \partial & 0 \\
0 & 0 & 0 & -1-i v \partial
\end{array}\right), \\
\theta_{2}^{\dagger} & =\left(\begin{array}{cccc}
\left(R_{3}\right)^{*} & r_{1} \partial^{-1} q_{2} & -2 r_{1} \partial^{-1} r_{1} & -r_{1} \partial^{-1} r_{2}-r_{2} \partial^{-1} r_{1} \\
r_{2} \partial^{-1} q_{1} & \left(R_{4}\right)^{*} & -r_{2} \partial^{-1} r_{1}-r_{1} \partial^{-1} r_{2} & -2 r_{2} \partial^{-1} r_{2} \\
-2 q_{1} \partial^{-1} q_{1} & -q_{1} \partial^{-1} q_{2}-q_{2} \partial^{-1} q_{1} & -R_{3} & q_{1} \partial^{-1} r_{2} \\
-q_{2} \partial^{-1} q_{1}-q_{1} \partial^{-1} q_{2} & -2 q_{2} \partial^{-1} q_{2} & q_{2} \partial^{-1} r_{1} & -R_{4}
\end{array}\right) .
\end{aligned}
$$

Introducing

$$
q_{i}=\sqrt{\gamma-i \mu v} \hat{q}_{i}, r_{i}=\sqrt{\gamma-i \mu v} \hat{r}_{i},(i=1,2),
$$

then from Eq. (4.2), we have

$$
\begin{aligned}
& \left(\begin{array}{cccc}
\hat{R}_{1}^{*} & \hat{r}_{1} \partial^{-1} \hat{q}_{2} & -2 \hat{r}_{1} \partial^{-1} \hat{r}_{1} & -\hat{r}_{1} \partial^{-1} \hat{r}_{2}-\hat{r}_{2} \partial^{-1} \hat{r}_{1} \\
\hat{r}_{2} \partial^{-1} \hat{q}_{1} & \hat{R}_{2}^{*} & -\hat{r}_{2} \partial^{-1} \hat{r}_{1}-\hat{r}_{1} \partial^{-1} \hat{r}_{2} & -2 \hat{r}_{2} \partial^{-1} \hat{r}_{2} \\
-2 \hat{q}_{1} \partial^{-1} \hat{q}_{1} & -\hat{q}_{1} \partial^{-1} \hat{q}_{2}-\hat{q}_{2} \partial^{-1} \hat{q}_{1} & -\hat{R}_{1} & \hat{q}_{1} \partial^{-1} \hat{r}_{2} \\
-\hat{q}_{2} \partial^{-1} \hat{q}_{1}-\hat{q}_{1} \partial^{-1} \hat{q}_{2} & -2 \hat{q}_{2} \partial^{-1} \hat{q}_{2} & \hat{q}_{2} \partial^{-1} \hat{r}_{1} & -\hat{R}_{2}
\end{array}\right) G_{\mu} \\
& =\frac{\mu}{\gamma-i \mu \nu}\left(\begin{array}{cccc}
1 & 0 & 0 & 0 \\
0 & 1 & 0 & 0 \\
0 & 0 & -1 & 0 \\
0 & 0 & 0 & -1
\end{array}\right) G_{\mu},
\end{aligned}
$$

where

$$
\hat{R}_{1}=\partial-2 \hat{q}_{1} \partial^{-1} \hat{r}_{1}-\hat{q}_{2} \partial^{-1} \hat{r}_{2}, \hat{R}_{2}=\partial-2 \hat{q}_{2} \partial^{-1} \hat{r}_{2}-\hat{q}_{1} \partial^{-1} \hat{r}_{1} .
$$

We observe that Eq. (4.3) enjoys the same structures as the eigenvalue equation (2.17). This observation implies the following linear spectral problem for the vFL equation

$$
\begin{aligned}
\Phi_{x} & =\left(\begin{array}{l}
\phi_{1} \\
\phi_{2} \\
\phi_{3}
\end{array}\right)_{x}=\left(\begin{array}{ccc}
2 \hat{\lambda} & \hat{q}_{1} & \hat{q}_{2} \\
\hat{r}_{1} & -\hat{\lambda} & 0 \\
\hat{r}_{2} & 0 & -\hat{\lambda}
\end{array}\right)\left(\begin{array}{l}
\phi_{1} \\
\phi_{2} \\
\phi_{3}
\end{array}\right) \\
& =\left(\begin{array}{ccc}
\frac{2 i\left(1-\gamma \xi^{2}\right)}{3 v} & \xi q_{1} & \xi q_{2} \\
\xi r_{1} & -\frac{i\left(1-\gamma \xi^{2}\right)}{3 v} & 0 \\
\xi r_{2} & 0 & -\frac{i\left(1-\gamma \xi^{2}\right)}{3 v}
\end{array}\right)\left(\begin{array}{l}
\phi_{1} \\
\phi_{2} \\
\phi_{3}
\end{array}\right)=M \Phi,
\end{aligned}
$$

where $\xi=\frac{1}{\sqrt{\gamma-i \mu \nu}}$ is the spectral parameter. Next we calculate the $t$-part of the spectral problem, which is assumed as

$$
\Phi_{t}=\left(\begin{array}{c}
\phi_{1} \\
\phi_{2} \\
\phi_{3}
\end{array}\right)_{t}=\left(\begin{array}{cccc}
-E & -K & A & B \\
C & E & F \\
G & H & K
\end{array}\right)\left(\begin{array}{l}
\phi_{1} \\
\phi_{2} \\
\phi_{3}
\end{array}\right)=N \Phi,
$$

where $A, B, C, E, F, G, H, K$ are functions depending on the field variables. 
Requiring that the zero curvature condition $M_{t}=N_{x}+[N, M]$ is equivalent to the vFL equation yields the following

$$
\begin{aligned}
A=-\frac{u_{1}}{v} \xi^{-1}+\frac{\gamma q_{1}}{v} \xi, & B=-\frac{u_{2}}{v} \xi^{-1}+\frac{\gamma q_{2}}{v} \xi, \\
C=-\frac{v_{1}}{v} \xi^{-1}+\frac{\gamma r_{1}}{v} \xi, & G=-\frac{v_{2}}{v} \xi^{-1}+\frac{\gamma r_{2}}{v} \xi, \\
F=i u_{2} v_{1}, \quad E & =i\left(\frac{1}{3 v^{2}} \xi^{-2}+u_{1} v_{1}-\frac{2 \gamma}{3 v^{2}}+\frac{\gamma^{2}}{3 v^{2}} \xi^{2}\right), \\
H=i u_{1} v_{2}, \quad K & =i\left(\frac{1}{3 v^{2}} \xi^{-2}+u_{2} v_{2}-\frac{2 \gamma}{3 v^{2}}+\frac{\gamma^{2}}{3 v^{2}} \xi^{2}\right) .
\end{aligned}
$$

Thus, we succeeded in finding the spectral problem Eqs. (4.4) and (4.5) for the Eq. (3.2).

It is known that the FL equation is a negative flow of the derivative nonlinear Schrödinger (DNLS) [15]. As a final observation, we comment on the relationship between the vFL equation and two component DNLS equation proposed by Morris and Dodd [23]. In fact, if we take

$$
\phi_{1}=e^{\frac{2 i x}{3 v}} \psi_{1}, \phi_{2}=e^{\frac{-i x}{3 v}} \psi_{2}, \phi_{3}=e^{\frac{-i x}{3 v}} \psi_{3}, \xi=\sqrt{\frac{3 v}{\gamma} \zeta},
$$

then the Eq. (4.4) becomes

$$
\left(\begin{array}{l}
\psi_{1} \\
\psi_{2} \\
\psi_{3}
\end{array}\right)_{x}=\left(\begin{array}{ccc}
-2 i \zeta^{2} & \alpha e^{\frac{-i x}{v}} \zeta q_{1} & \alpha e^{\frac{-i x}{v}} \zeta q_{2} \\
\alpha e^{\frac{i x}{v} \zeta r_{1}} & i \zeta^{2} & 0 \\
\alpha e^{\frac{i x}{v} \zeta r_{2}} & 0 & i \zeta^{2}
\end{array}\right)\left(\begin{array}{l}
\psi_{1} \\
\psi_{2} \\
\psi_{3}
\end{array}\right)
$$

where $\alpha=\sqrt{\frac{3 v}{\gamma}}$. The same transformation converts the Eq. (4.5) into

$$
\Phi_{t}=\left(\begin{array}{l}
\psi_{1} \\
\psi_{2} \\
\psi_{3}
\end{array}\right)_{t}=\left(\begin{array}{ccc}
-\tilde{E}-\tilde{K} & e^{\frac{-i x}{v}} \tilde{A} & e^{\frac{i x}{v}} \tilde{B} \\
e^{\frac{i x}{v}} \tilde{C} & \tilde{E} & \tilde{F} \\
e^{\frac{i x}{v}} \tilde{G} & \tilde{H} & \tilde{K}
\end{array}\right)\left(\begin{array}{l}
\psi_{1} \\
\psi_{2} \\
\psi_{3}
\end{array}\right)
$$

where

$$
\begin{aligned}
& \tilde{A}=-\frac{u_{1}}{\alpha v} \zeta^{-1}+\frac{\gamma q_{1} \alpha}{v} \zeta, \tilde{B}=-\frac{u_{2}}{\alpha v} \zeta^{-1}+\frac{\gamma q_{2} \alpha}{v} \zeta, \\
& \tilde{C}=-\frac{v_{1}}{\alpha \nu} \zeta^{-1}+\frac{\gamma r_{1} \alpha}{v} \zeta, \tilde{G}=-\frac{v_{2}}{\alpha v} \zeta^{-1}+\frac{\gamma r_{2} \alpha}{v} \zeta, \\
& \tilde{F}=i u_{2} v_{1}, \quad \tilde{E}=i\left(\frac{1}{3 v^{2} \alpha^{2}} \zeta^{-2}+u_{1} v_{1}-\frac{2 \gamma}{3 v^{2}}+\frac{\gamma}{v} \zeta^{2}\right), \\
& \tilde{H}=i u_{1} v_{2}, \quad \tilde{K}=i\left(\frac{1}{3 v^{2} \alpha^{2}} \zeta^{-2}+u_{2} v_{2}-\frac{2 \gamma}{3 v^{2}}+\frac{\gamma}{v} \zeta^{2}\right) .
\end{aligned}
$$

Now it is easy to see that (4.7) is nothing but the spatial part of the Lax pair for the two component DNLS hierarchy [23] (see also Ref. [18]). Therefore, the vFL system may be regarded as a particular (negative) flow of the two component DNLS hierarchy. 


\section{Acknowledgments}

We would like to thank Q. P. Liu, LingLing Xue and NianHua Li for interesting discussions and suggestions. The helpful comments of the anonymous referee have been taken accounted in revising the paper. This work is supported by the National Natural Science Foundation of China (grant numbers: 11401572, 10971222 and 11271366), the Fundamental Research Funds for Central Universities and "New Start" Academic Research Projects of Beijing Union University (grant numbers: ZK10201412).

\section{References}

[1] M. J. Ablowitz, Nonlinear Dispersive Waves: Asymptotic Analysis and Solitons, Cambridge Texts in Appl. Math. 47 (Cambridge University Press, Cambridge, England, 2011).

[2] G. P. Agrawal, Nonlinear Fiber Optics, 4th edition (Academic Press, Boston, 2007).

[3] H. Aratyn, J. F. Gomes, and A. H. Zimerman, Affine Lie algebraic origin of constrained KP hierarchies, J. Math. Phys. 36 (1995) 3419-3442.

[4] Y. Cheng, Constraints of the KP hierarchy, J. Math. Phys. 33 (1992) 3774-3782.

[5] Y. Cheng and Y. S. Li, The constraint of the Kadomtsev-Petviashvili equation and its special solutions, Phys. Lett. A 157 (1991) 22-26.

[6] A. S. Fokas, On a class of physically important integrable equations, Physica D 87 (1995) 145-150.

[7] A. S. Fokas and R. L. Anderson, On the use of isospectral eigenvalue problems for obtaining hereditary symmetries for Hamiltonian systems, J. Math. Phys. 23 (1982) 1066-1073.

[8] A. S. Fokas and B. Fuchssteiner, On the structure of symplectic operators and hereditary symmetries, Lett. Nuovo Cimento 28 (1980) 299-303.

[9] A. P. Fordy and P. P. Kulish, Nonlinear Schrödinger equations and simple Lie algebras, Commun. Math. Phys. 89 (1983) 427-443.

[10] B. Fuchssteiner, Application of spectral-gradient methods to nonlinear soliton equations (unpublished, http://fuchssteiner.info/papers/29.pdf).

[11] B. Fuchssteiner, Some tricks from the symmetry-toolbox for nonlinear equations: generalizations of the Camassa-Holm equation, Physica D 95 (1996) 229-243.

[12] J. He, S. Xu and K. Porsezian, Rogue waves of the Fokas-Lenells equation, J. Phys. Soc. Jpn. 81 (2012) 124007.

[13] B. G. Konopelchenko, J. Sidorenko and W. Strampp, (1+1)-dimensional integrable systems as symmetry constraints of (2+1)-dimensional systems, Phys. Lett. A 157 (1991) 17-21.

[14] B. G. Konepelchenko and W. Strampp, The AKNS hierarchy as symmetry constraint of the KP hierarchy, Inv. Prob. 7 (1991) 417-429.

[15] J. Lenells, Exactly solvable model for nonlinear pulse propagation in optical fibers, Stud. Appl. Math. 123 (2009) 215-232.

[16] J. Lenells, Dressing for a novel integrable generalization of the nonlinear Schrödinger equation, $J$. Nonlinear Sci. 20 (2010) 709-722.

[17] J. Lenells and A. S. Fokas, On a novel integrable generalization of the nonlinear Schrödinger equation, Nonlinearity 22 (2009) 11-27.

[18] L. Ling and Q. P. Liu, Darboux transformation for a two-component derivative nonlinear Schrödinger equation, J. Phys. A: Math. Theor. 43 (2010) 434023.

[19] Q. P. Liu, Bi-Hamiltonian structures of the coupled AKNS hierarchy and the coupled Yajima Oikawa hierarchy, J. Math. Phys. 37 (1996) 2307-2314.

[20] S. V. Manakov, On the theory of two-dimensional stationary self-focusing of electromagnetic waves, Sov. Phys. JETP 38 (1974) 248-263.

[21] Y. Matsuno, A direct method of solution for the Fokas-Lenells derivative nonlinear Schrödinger equation: I. bright soliton solutions, J. Phys. A: Math. Theor. 45 (2012) 23202.

[22] Y. Matsuno, A direct method of solution for the Fokas-Lenells derivative nonlinear Schrödinger equation: II. dark soliton solutions, J. Phys. A: Math. Theor. 47 (2012) 475202. 
[23] H. C. Morris and R. K. Dodd, The two component derivative nonlinear Schrödinger equation, Phys. Scr. 20 (1979) 505-508.

[24] P. J. Olver and P. Rosenau, Tri-Hamiltonian duality between solitons and solitary-wave solutions having compact support, Phys. Rev. E 53 (1996) 1900-1906.

[25] J. Sidorenko and W. Strampp, Symmetry constraints of the KP hierarchy, Inv. Prob. 7 (1991) L37-L44.

[26] V. E. Vekslerchik, Lattice representation and dark solitons of the Fokas-Lenells equation, Nonlinearity 24 (2011) 1165-1175.

[27] J. Yang, Nonlinear Waves in Integrable and Nonintegrable Systems ( SIAM, 2010).

[28] P. Zhao, E. Fan and Y. Hou, Algebro-geometric solutions and their reductions for the Fokas-Lenells hierarchy (http://arxiv.org/abs/1305.0122v2). 